\title{
Gerenciamento de resíduos sólidos em unidade de terapia intensiva
}

\author{
José Dionísio de Paula Júnior*, Sandra Maria Jannotti Quintão**, \\ Maria Augusta Coutinho de Andrade Oliveira***, Larissa Rosa da Neiva Sampaio****, \\ Letícia Moreira Carlos****
}

*Enfermeiro do Trabalho, Docente da Fundação Presidente Antônio Carlos - FUPAC/Ubá, Doutorando em Ciências Biomédicas IUNIR, Argentina, **Enfermeira especialista em Educação Profissional pela FIOCRUZ, Coordenadora do Serviço de Controle de Infecção Hospitalar da Casa de Saúde Padre Damião (FHEMIG), docente da Fundação Presidente Antônio Carlos - FUPACl Ubá, ***Enfermeira Responsável Técnica pela Irmandade Nossa Senhora da Saúde Hospital São Vicente de Paula, Especialista em Gestão Hospitalar. Docente da Fundação Presidente Antônio Carlos - FUPAC/Ubá, ****Curso de Enfermagem da Fundação Presidente Antônio Carlos - FUPAC/Ubá

\section{Resumo}

Objetivo: Descrever o processo de acondicionamento de resíduos sólidos do serviço de saúde (RSSS) produzidos em unidades de terapia intensiva (UTI). Material e métodos: Pesquisa descritiva exploratória com temporalidade transversal. O cenário foi a Unidade de Terapia Intensiva (UTI) de um hospital filantrópico do município de Ubá/MG. As informaçóes do acondicionamento dos RSSS foram registradas por câmera digital da marca Nikon’, por acadêmicas do curso de graduação de Enfermagem. Resultados: São produzidos no setor resíduos que pertencem aos grupos A, D e E, identificados por nomes e símbolos padronizados pela instituição. Foram identificados resíduos comuns acondicionados junto aos infectantes. Conclusão: A pesquisa mostrou a existência de falhas no acondicionamento dos resíduos sólidos gerados na UTI.

Palavras-chave: gerenciamento, resíduos, unidade de tratamento intensivo.

\section{Abstract \\ Solid waste management in the intensive care unit}

Objective: To describe packaging process of solid health service waste (SHSW) produced in intensive care units (ICU). Methods: This was a descriptive and exploratory research with transverse temporality. The scenario was the Intensive Care Unit (ICU) of a philanthropic hospital in the city of Ubá/MG. Data concerning packaging of SHSW were recorded by Nikon digital camera by students of undergraduate nursing course. Results: The packaging was carried out in the solid waste management department that belongs to groups A, D and E, identified by names and standardized symbols. We identified common waste packaged next to infectious waste. Conclusion: The research showed that there was packaging failures of solid waste generated in the ICU.

Key-words: management, waste, intensive care unit. 


\section{Resumen}

\section{Gestión de residuos sólidos en la unidad de cuidados intensivos}

Objetivo: Describir el proceso de almacenamiento de residuos sólidos de servicios de salud (RSSS) en la Unidad de Cuidados Intensivos (UCI). Materiales y métodos: Se trata de investigación descriptiva y exploratorio con temporalidad transversal. El escenario fue la Unidad de Cuidados Intensivos (UCI) en hospital de la ciudad de Ubá, Minas Gerais. Los datos de imágenes referente al acondicionamiento fueron creados con cámara digital Nikon', por estudiantes del curso de graduación en enfermería. Resultados: Se producen en el sector los residuos que pertenecen a grupos A, D y E, identificados por sus nombres y símbolos estándar. Hemos identificado residuos comunes depositados junto a los infecciosos. Conclusión: La investigación ha demostrado la existencia de fallas en la separación de los residuos sólidos generados en la UCI.

Palabras-clave: manejo, residuos, unidad de cuidados intensivos.

\section{Introdução}

Os Resíduos de Serviços de Saúde (RSS) são definidos conforme a Resolução Conselho Nacional do Meio Ambiente 283/2001 (CONAMA) como aqueles provenientes de qualquer unidade que execute atividades de natureza médica, assistencial, humana e animal, os provenientes de centros de pesquisa, desenvolvimento ou experimentação na área de farmacologia e saúde, medicamentos e imunoterápicos vencidos ou deteriorados, aqueles provenientes de necrotérios, funerárias, serviços de medicina legal e aqueles provenientes de barreiras sanitárias [1-5].

Para que haja um controle sobre o gerenciamento destes RSS, cada unidade geradora deve elaborar um Plano de Gerenciamento de Resíduos de Serviços de Saúde (PGRSS), seguindo normas e regulamentos exigidos pela Agência Nacional de Vigilância Sanitária (Anvisa). Neste caso, conhecer os procedimentos para um gerenciamento adequado dos RSS representa desafios a serem enfrentados pelas equipes de saúde [6].

O PGRSS deve ser constituído de procedimentos de gestáo, com o objetivo de minimizar a produção de resíduos e proporcionar um encaminhamento seguro e eficiente, visando à proteção dos trabalhadores, a preservação da saúde pública, dos recursos naturais e do meio ambiente. Este plano é estabelecido nas seguintes etapas: segregação, que consiste na separaçáo dos resíduos no momento e local de sua geraçáo de acordo com suas características; acondicionamento, que é o ato de embalar os resíduos segregados corretamente, em sacos e/ ou recipientes impermeáveis, resistentes à ruptura e vazamentos, e sua capacidade deve ser compatível com sua geração diária; identificação, os resíduos devem ser identificados por símbolos, cores e frases diferentes de acordo com sua classificação, atendendo as normas da Associação Brasileira de Normas Técnicas (ABNT); transporte interno, que consiste no transporte do local da sua geraçáo até o local de armazenamento temporário, ou a apresentação para coleta externa que deve ser realizada separadamente e em recipientes específicos a cada grupo de resíduos; armazenamento temporário, onde ocorre a guarda temporária dos recipientes contendo os resíduos já acondicionados, em local próximo aos pontos de geração, visando agilizar a coleta dentro do estabelecimento; tratamento, que consiste na aplicação de processo que modifique as características biológicas ou a composição dos RSS, que leve à redução ou eliminaçáo dos riscos de causar doenças; armazenamento externo, que acondiciona os resíduos até ser realizada a sua coleta externa, com acesso facilitado para os veículos coletores; coleta e transporte externos, onde ocorre à remoção dos resíduos até a unidade de tratamento ou destinação final; destino final, onde há disposição de resíduos no solo [6,7].

Os resíduos são classificados em cinco grupos (A, B, C, D e E): No grupo A, são resíduos com presença de agentes infectantes, que são acondicionados em saco plástico branco-leitoso, e seu tratamento consiste na incineração, que é a queima dos resíduos em fornos ou usinas próprias. No grupo B, consistem os resíduos contendo substâncias químicas, que são acondicionados dentro de sua própria embalagem, envolvidos por um saco e devolvidos ao fabricante. No grupo C, são resíduos resultantes de atividade humana que contenham rejeitos radioativos, que são descartados de acordo com normas da Comissão Nacional de Energia Nuclear - CNEN. O grupo $\mathrm{D}$, pode ser equiparado com o lixo domiciliar, seu acondicionamento ocorre em saco plástico preto, 
sendo reutilizados, reciclados ou aterrados. O grupo E, são os resíduos perfurocortantes ou escarificantes, que devem ser acondicionados em recipientes rígidos e incinerados $[7,8]$.

As falhas ocorridas no processo de gerenciamento de resíduos acarretam em riscos para a saúde ambiental, porém, estes riscos podem ser minimizados pelo gerenciamento adequado e um destino final seguro destes resíduos [9-13].

Observa-se que o gerenciamento de resíduos em unidades de terapia intensiva (UTI) precisa seguir padróes rigorosos na prevenção de riscos a saúde do trabalhador e impactos para a saúde pública.

É de grande importância estabelecer, por meio de investigação científica, a relação entre o manuseio dos resíduos sólidos de serviços de saúde (RSSS) e os riscos para saúde do trabalhador em instituição hospitalar.

Esta pesquisa tem como objetivo descrever o processo de acondicionamento de resíduos sólidos do serviço de saúde produzidos em unidades de terapia intensiva.

\section{Material e métodos}

Esta pesquisa é um estudo descritivo exploratório com temporalidade transversal. A pesquisa aconteceu no período de setembro e outubro de 2011 . O cenário foi um hospital filantrópico do município de Ubá, Minas Gerais. O local investigado foi a Unidade de Terapia Intensiva (UTI), constituído por 9 leitos e 18 funcionários, composto pela equipe de enfermagem, médicos e profissionais da limpeza.

A coleta de dados ocorreu por acadêmicas do curso de graduação em Enfermagem da Fundação Presidente Antônio Carlos (FUPAC), em dias que os funcionários não conheciam, a fim de prevalecer maior fidedignidade da amostra. A investigação aconteceu entre os horários de 7:00 h as 19:00 h, após levantamento de horários preconizados pela instituição para o gerenciamento de resíduos.

As informações do acondicionamento dos RSSS foram registradas por câmera digital da marca Nikon ${ }^{\oplus}$, pelas próprias acadêmicas do curso de graduação.

Método de inclusão foram os resíduos sólidos, resíduos gerados na UTI; resíduos gerados no dia do registro de imagens. Método de exclusão foram os resíduos líquidos.

O trabalho obedeceu todos os critérios éticos em acordo com a resoluçáo 196/96 do Conselho
Nacional de Saúde e foi aprovado pelo comitê de ética e pesquisa da FUPAC (02/011).

Para análises dos dados realizou-se a relação das imagens obtidas com as normas técnicas empregadas para o acondicionamento dos RSSS, fundamentado na RDC Anvisa [6]

\section{Resultados}

São produzidos no setor, resíduos que pertencem aos grupos A, D e E, identificados por nomes e símbolos padronizados pela instituição (Figura 1).

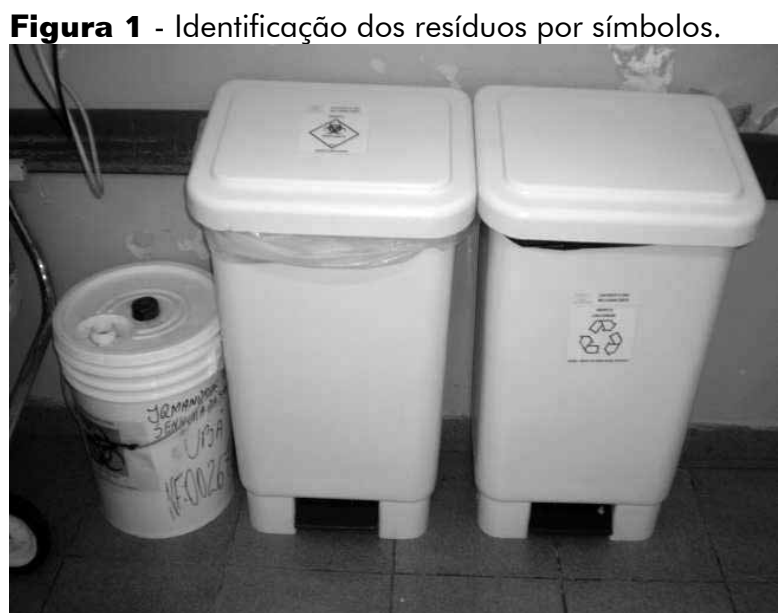

O grupo A deveria estar acondicionado em saco plástico branco, em recipientes rígidos, com pedal e tampa, pois foram encontrados resíduos comuns acondicionados junto aos infectantes, como copos descartáveis e papeis toalha (Figura 2).

Figura 2 - Acondicionamento de resíduos infectantes e comuns.

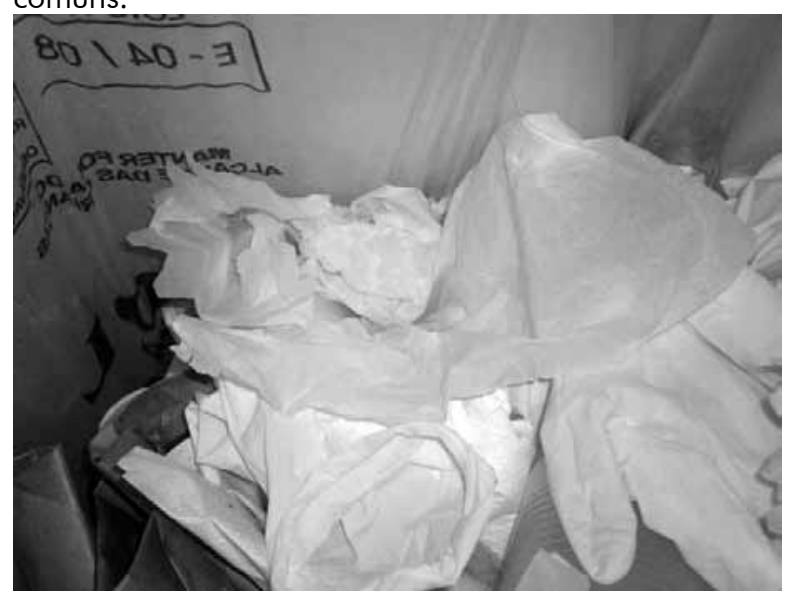

Lixeira de resíduos infectantes apresentou em seu envoltório interno saco plástico preto, que é referente ao do grupo D (Figura 3). 
Figura 3 - Lixeira de resíduos infectantes.

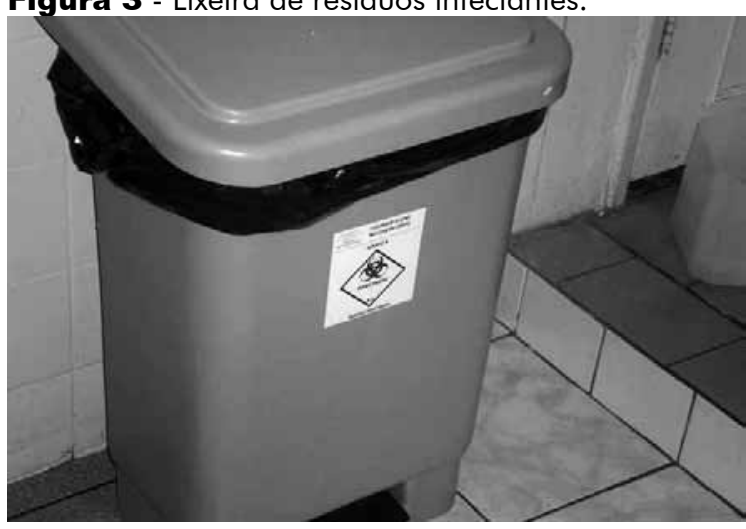

A lixeira do grupo D, no momento da pesquisa, ultrapassava sua capacidade máxima permitida (Figura 4).

Figura 4 - Lixeira de resíduos comuns.

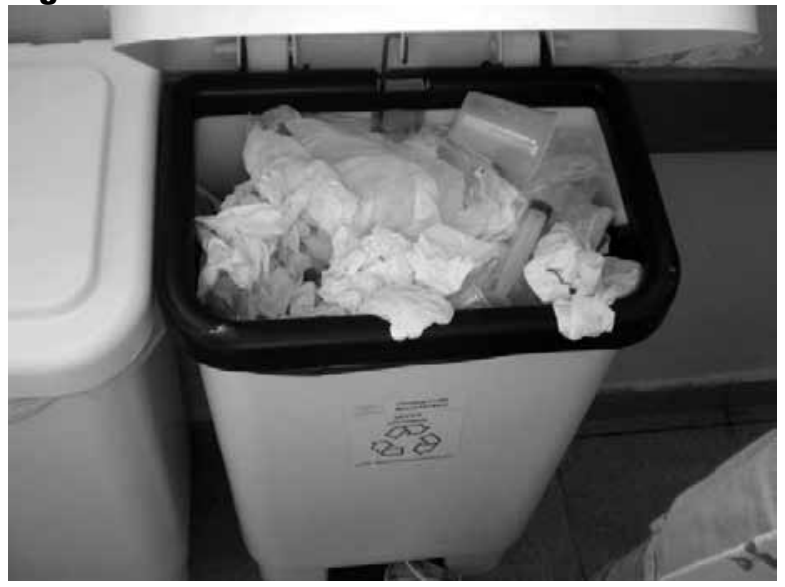

Os resíduos do grupo $\mathrm{E}$ foram acondicionados em recipiente rígido, resistente à ruptura, punctura e vazamento, como determina a legislação, ressaltado que dentro deste recipiente havia também o descarte de resíduos do grupo $\mathrm{D}$, como: embalagens de seringas e papeis no geral (Figura 5).

Figura 5 - Acondicionamento dos perfuro cortantes.

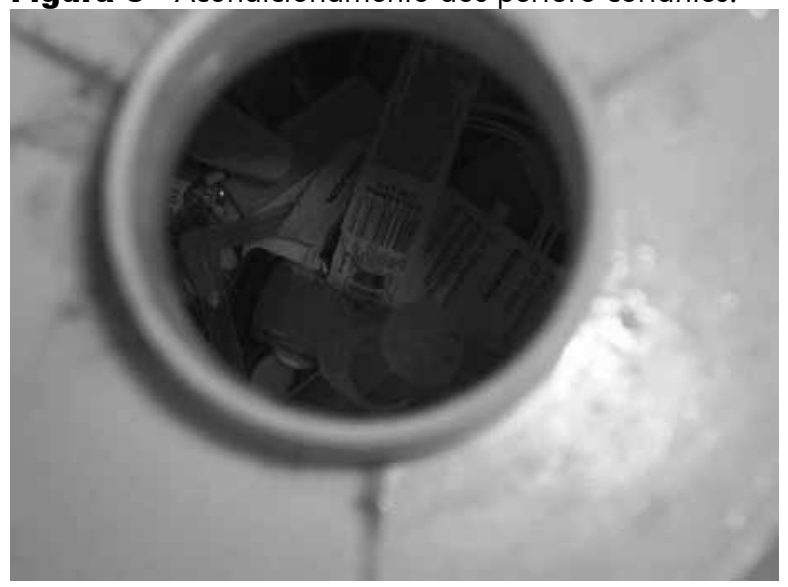

Os resíduos gerados na UTI eram recolhidos de acordo com a atividade diária do setor. $\mathrm{O}$ transporte interno de resíduos deve ser realizado atendendo um roteiro previamente definido e em horários não coincidentes com a distribuição de roupas, alimentos e medicamentos, períodos de visita ou de maior fluxo de pessoas ou de atividades.

O carro para o transporte interno apresentava todos os padróes necessários para atividade, no setor pesquisado, entretanto transportava todos os grupos (A, D e E) simultaneamente.

\section{Discussão}

Analisando as informaçóes obtidas por foto imagem dos resíduos da UTI, e em acordo com a norma NBR 7.500 da ABNT [14], os RSS devem ser identificados para permitir fácil visualização, de forma indelével, utilizando símbolos, cores e frases, atendendo as características de cada resíduo. O estudo de Lopes et al. [15] possibilita refletir sobre os problemas referente aos procedimentos de enfermagem. A escassez de recursos, materiais de baixa qualidade, relacionando ainda com o pouco incentivo para os profissionais de enfermagem, podem refletir em um gerenciamento de resíduos em UTI de baixa qualidade.

De acordo com a NBR 12.807/93 [14], a segregação, primeira fase do gerenciamento, diz respeito ao ato de separar os resíduos no momento de sua geração, segundo a classificação previamente estabelecida. Para Lopes et al. [16], a segregação do RSSS por grupos, possibilitaria o melhor manejo e destino para cada tipo de resíduo, reduzindo custos e permitindo inclusive a implantaçáo do sistema de coleta seletiva e reciclagem.

Em estudos de Macedo et al. [17], sobre a segregação dos serviços de saúde, foram encontradas algumas falhas na segregação envolvendo resíduos dos grupo A. Almeida et al. [9] recomendam que o acondicionamento dos resíduos nos serviços de saúde ocorra em sacos plásticos resistentes ou em recipientes adequados, rígidos e que náo exceda a dois terços do total de sua capacidade mínima. Os autores alertam ainda sobre o elevado risco infectante que esta classe de resíduo apresenta [9].

Em pesquisa realizada com profissionais de enfermagem em hospital universitário, Canini et al. [18] preconizam a importância da conscientização da equipe de enfermagem, quanto à necessidade de descartar os materiais perfurocortantes em local 
adequado, que influencia diretamente na reduçáo de acidentes de trabalho.

Segundo Takada [19], metade dos leitos ocupados por pacientes que contraíram alguma infecção hospitalar foi associado a problemas de saneamento, higiene do ambiente, instalaçóes inadequadas e negligência dos profissionais de Enfermagem ao manipular materiais exercendo os cuidados em locais impróprios. Expóem também, que o manejo inadequado dos resíduos é responsável, direta ou indiretamente, por $10 \%$ das enfermidades adquiridas pelos pacientes, durante o período de internação hospitalar [17]. Entretanto Naime et al. [20] frisam a importância de educação em biossegurança com a capacitação dos profissionais expostos aos RSS, a fim de preservar a integridade desses profissionais e a conservação da saúde dos pacientes internados.

Os carros para transporte interno devem ser constituídos de material rígido, lavável, impermeável, resistente ao processo de descontaminação, provido de tampa articulada ao próprio corpo do equipamento, cantos e bordas arredondados, e identificados com o símbolo correspondente ao risco do resíduo neles contidos [6]. Segundo Almeida et al. [9],esse transporte deve ser realizado em sentido único e separadamente para cada tipo de resíduo.

\section{Conclusão}

A pesquisa mostrou a existência de falhas no acondicionamento dos resíduos sólidos gerados na UTI. O cumprimento adequado de todas as etapas do manejo interno dos RSSS representa diminuição de riscos para a saúde do trabalhador e saúde ambiental.

\section{Referências}

1. Feitosa LJ, Antunes JRP, Aranda AC. Boas práticas em resíduos de serviços de saúde (RSS). Rio de Janeiro: Secretaria do Estado do Rio de Janeiro; 2002. p.1-15.

2. Paveloski EM, Hamaba J. Segregação dos resíduos de serviços de saúde como processo de produçáo mais limpa: estudo de caso da $7^{\text {a }}$ Região administrativa do Estado de Sáo Paulo. 2009. [citado 2012 Jan 25]. Disponível em: http://www.advancesincleanerproduction. net/second/files/sessoes

3. Zamoner M. Modelo para avaliação de planos de gerenciamento de resíduos de serviços de saúde (PGRSS) para secretarias municipais da saúde e/ou do meio ambiente. Ciênc Saúde Coletiva 2008;13(6):1945-52.

4. Sales CCL, Spolti GP, Lopes MSB, Lopes DF. Gerenciamento dos resíduos sólidos dos serviços de saúde: aspectos do manejo interno no município de Marituba, Pará, Brasil. Ciênc Saúde Coletiva 2009;14(6):31-8.
5. Silva CE, Hoppe AE. Diagnóstico dos resíduos de serviços de saúde no interior do Rio Grande do Sul. Eng Sanit Ambient 2005;10(2):146-51.

6. Ministério da saúde. Agência Nacional de Vigilância Sanitária (ANVISA). Resolução - RDC no 306, de 7 de dezembro de 2006. Dispóe sobre o Regulamento Técnico para o gerenciamento de resíduos de serviços de saúde. Brasília: MS; 2006.

7. Garcia LP, Zanetti-Ramos BG. Gerenciamento dos resíduos de serviços de saúde: uma questão de biossegurança. Cad Saúde Pública 2004;20(3):744-52.

8. Tramontini A, Pandolfo A, Guimarães J, Reinehr R, Oliveira CRR, Pandolfo LM. Resíduos de serviços de saúde: uma abordagem prática em hospitais gerais da cidade de Passo Fundo. Revista Saúde e Ambiente 2009;10(2):45-53.

9. Almeida VCF, Pinto SL, Nascimento AJR, Feitosa CR, Alencar PRP. Gerenciamento dos resíduos sólidos em unidades de saúde da família. Rev RENE 2009;10(2):103-12.

10. Naime R, Ramalho AHP, Naime IS. Avaliação do sistema de gestáo dos resíduos sólidos do hospital de clínicas de Porto Alegre. Revista Espaço para a Saúde 2008;9(1):1-17.

11. Takahashi RT, Gonçalves VLM. Gerenciamento de recursos físicos e ambientais. In: Kurcgant P, Tronchin DMR, Fugulin FMT, Peres HHC, Massarollo MCKB, Fernandes MFP, et al. Gerenciamento em enfermagem. Rio de Janeiro: Guanabara Koogan 2005;1(1):184-94.

12. Corrêa LB, Lunardi VL, Conto SMD. O processo de formação em saúde: o saber resíduos sólidos de serviços de saúde em vivências práticas. Rev Bras Enferm 2007;60(1):21-5.

13. Oliveira JM. Análise do Gerenciamento de Resíduos de Serviços de Saúde nos hospitais de Porto Alegre. Porto Alegre: Universidade Federal do Rio Grande do Sul 2002; p.1-96.

14. Ministério da Saúde. Agência Nacional de Vigilância Sanitária (ANVISA), Resolução - RDC n³3, de 25 de fevereiro de 2003. Dispóe sobre o Regulamento técnico para o gerenciamento de resíduos de serviços de saúde. Brasília: MS; 2003.

15. Lopes L, Dyniewicz A, Kalinowski L. Gerenciamento de Materiais e custos Hospitalar em UTI Neonatal. Cogitare Enferm 2010;15(2):278-85.

16. Lopes LKO, Tipple AFV, Damando SN, Miranda CS, Gomes IV. Atendimento aos profissionais vítimas de acidentes com material biológico em um hospital de doenças infectocontagiosas. Rev Eletrônica de Enferm 2004;06(03):324-9.

17. Macedo L, Larocca L, Chaves M, Perna P, Muntsch S, Damaceno C, et al.,Segregação de resíduos nos serviços de saúde - a educação ambiental em um hospital - escola. Cogitare Enferm 2007;12(2):183-8.

18. Canini SRMS, Hayashida M, Machado AA. Acidentes perfurocortantes entre trabalhadores de enfermagem de um hospital universitário do interior paulista. Rev Latinoam Enferm 2002;10(2):172-8.

19. Takada ACS. O plano de gerenciamento de resíduos de serviços de saúde e o direito do trabalhador. Brasília: Escola Nacional de Saúde Pública; 2003. p.1-35.

20. Naime R, Sartor I, Garcia AC. Uma abordagem sobre a gestão dos resíduos de serviços de saúde. Revista Espaço para a Saúde 2004;5(02):17-27. 